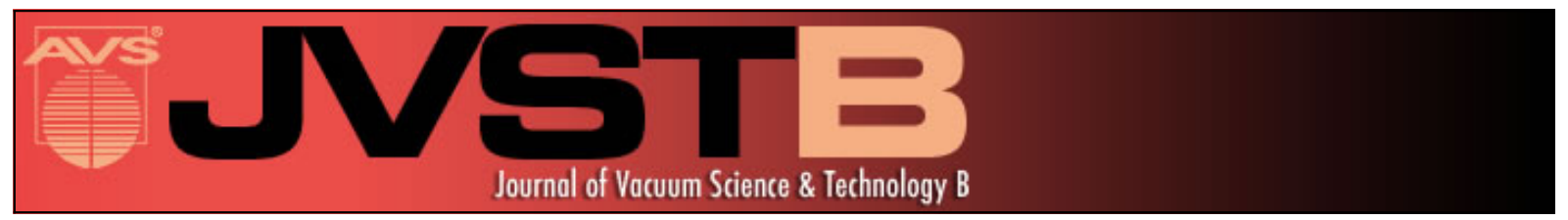

\title{
Reflection high-energy electron diffraction during substrate rotation: A new dimension for in situ characterization
}

W. Braun, H. Möller, and Y.-H. Zhang

Citation: Journal of Vacuum Science \& Technology B 16, 1507 (1998); doi: 10.1116/1.589976

View online: http://dx.doi.org/10.1116/1.589976

View Table of Contents: http://scitation.aip.org/content/avs/journal/jvstb/16/3?ver=pdfcov

Published by the AVS: Science \& Technology of Materials, Interfaces, and Processing

\section{Articles you may be interested in}

Reflection high-energy electron diffraction and scanning tunneling microscopy study of $\ln P(001)$ surface reconstructions

J. Vac. Sci. Technol. A 18, 1492 (2000); 10.1116/1.582373

Reflection high-energy electron diffraction oscillations on rotating substrates

J. Vac. Sci. Technol. B 17, 474 (1999); 10.1116/1.590579

New model for reflection high-energy electron diffraction intensity oscillations

J. Vac. Sci. Technol. B 16, 2404 (1998); 10.1116/1.590182

Reflection high-energy electron diffraction studies of wurtzite GaN grown by molecular beam epitaxy

J. Vac. Sci. Technol. B 16, 2237 (1998); 10.1116/1.590155

Molecular-beam epitaxy of BeTe layers on GaAs substrates studied via reflection high-energy electron diffraction Appl. Phys. Lett. 72, 2859 (1998); 10.1063/1.121482

\section{HDDEN}

Contact Hiden Analytical for further details: w www.HidenAnalytical.com E info@hiden.co.uk CLICK TO VIEW our product catalogue

\section{Instruments for Advanced Science}

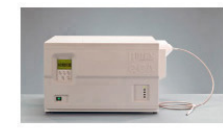

Gas Analysis

dynamic measurement of reaction gas streams catalysis and thermal analysis

molecular beam studies

, fermentation environmental and ecological studies

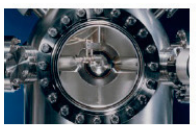

Surface Science UHVTPD

SIMS

end point detection in ion beam etch elemental imaging - surface mapoing

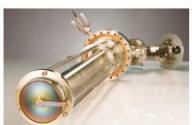

Plasma Diagnostics plasma source characterization etch and deposition process reaction kinetic studies analysis of neutral and radical species

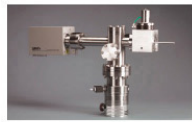

Vacuum Analysis - partial pressure measurement and control of process gases reactive sputter process control vacuum diagnostics vacuum coating process monitoring 


\title{
Reflection high-energy electron diffraction during substrate rotation: A new dimension for in situ characterization
}

\author{
W. Braun ${ }^{\mathrm{a})}$ \\ Center for Solid State Electronics Research and Department of Electrical Engineering, \\ Arizona State University, Tempe, Arizona 85287-6206 \\ H. Möller \\ Fraunhofer Institut für Integrierte Schaltungen-A, D-91058 Erlangen, Germany \\ Y.-H. Zhang \\ Center for Solid State Electronics Research and Department of Electrical Engineering, \\ Arizona State University, Tempe, Arizona 85287-6206
}

(Received 5 October 1997; accepted 12 December 1997)

\begin{abstract}
We present two methods to access reciprocal space with reflection high-energy electron diffraction (RHEED) during substrate rotation. The extraction of an arbitrary number of still frames from a continuously changing RHEED pattern is realized by triggering the substrate rotation and it allows analysis of quasistatic RHEED patterns that are updated every revolution. At the same time, the intensity along a line parallel to the shadow edge can be used to reconstruct a planar cut through the reciprocal lattice similar to a low-energy electron diffraction pattern. This RHEED pattern directly reveals the symmetry of the surface reconstruction and its changes during the deposition process.

(c) 1998 American Vacuum Society. [S0734-211X(98)02503-7]
\end{abstract}

\section{INTRODUCTION}

Although almost every molecular beam epitaxy (MBE) machine is equipped with a reflection high-energy electron diffraction (RHEED) system, the information contained in the diffraction pattern is generally not used during sample rotation. This limits the use of RHEED as a true in situ technique. Most layers for device or scientific applications have to be grown with substrate rotation because layer uniformity is of paramount importance. It is therefore highly desirable to develop methods that allow access to the reciprocal lattice of rotating substrates.

Several approaches using RHEED during rotation have focused on the specular spot shape and intensity during substrate rotation. ${ }^{1-4}$ The results demonstrate that it is possible to extract the growth rate by extracting the RHEED oscillation signal. These approaches are either based on intensity measurements combined with Fourier filtering, ${ }^{1,5}$ and high frequency rotation ${ }^{2}$ or measurements of the specular spot size combined with Fourier filtering. ${ }^{3}$ Even if the RHEED oscillations are not evaluated, the specular spot intensity varies depending on the growth conditions used and can be used to characterize MBE growth. ${ }^{4}$

RHEED measurements on nonrotating substrates, apart from growth rate determination ${ }^{6}$ and characterization of interfaces ${ }^{7}$ using RHEED oscillations, also use the information contained in the geometry and shape of the diffraction streaks to assess the surface structure in MBE. ${ }^{8}$ In this work, we examine ways of accessing this diffraction geometry information during substrate rotation. We demonstrate two methods that exploit the rotating substrate to obtain reciprocal lattice scans that are impossible with a static RHEED experiment. These new modes of operation expand the capa-

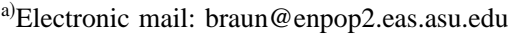

bilities of RHEED beyond the scope of traditional RHEED experiments and allow assessment of almost the entire accessible reciprocal space.

\section{EXPERIMENT}

The experiments were performed using a DCA MBE system equipped with a low wobble substrate manipulator, a standard RHEED e-gun operated at $20 \mathrm{kV}$ and a P20 phosphorus screen. Intensities were recorded at a rate of $50 \mathrm{~Hz}$ using a charge coupled device (CCD) camera and PC-based analysis system. ${ }^{9}$ A straightforward way to obtain static RHEED images from a rotating substrate is a triggered collection of images along high symmetry azimuths during rotation. The CCD camera taking images of the RHEED screen and the rotation motor are therefore interlocked via trigger signals. Images can then be extracted at arbitrary azimuths during the continuous rotation. The windows displaying the results for the different azimuths are updated once per revolution, allowing a quasisimultaneous display of the different quasistatic displays. An example of the implementation in Fig. 1 shows the capture of the four in-plane $\langle 110\rangle$ azimuths of a (001) GaAs $\beta(2 \times 4)$ reconstructed surface at $580^{\circ} \mathrm{C}$. The source area on the live image is marked by a bright frame on the continuous video image in the background. While the two [110] azimuths exhibiting the $2 \times$ pattern (left) are almost identical, the position of the specular spot in the two patterns along the [110] azimuth $(4 \times$, right) is different, indicating substrate wobble with a misorientation towards $[1 \overline{1} 0]$. For the $20 \mathrm{keV}$ electrons used in this measurement the distance between the (00) and (01) streaks corresponds to an angle of $1.23^{\circ}$. This yields a value of $0.5^{\circ}$ for the substrate wobble. The cyclic recording of images along several azimuths corresponds to the situation of several RHEED guns installed at the corresponding angles. It 


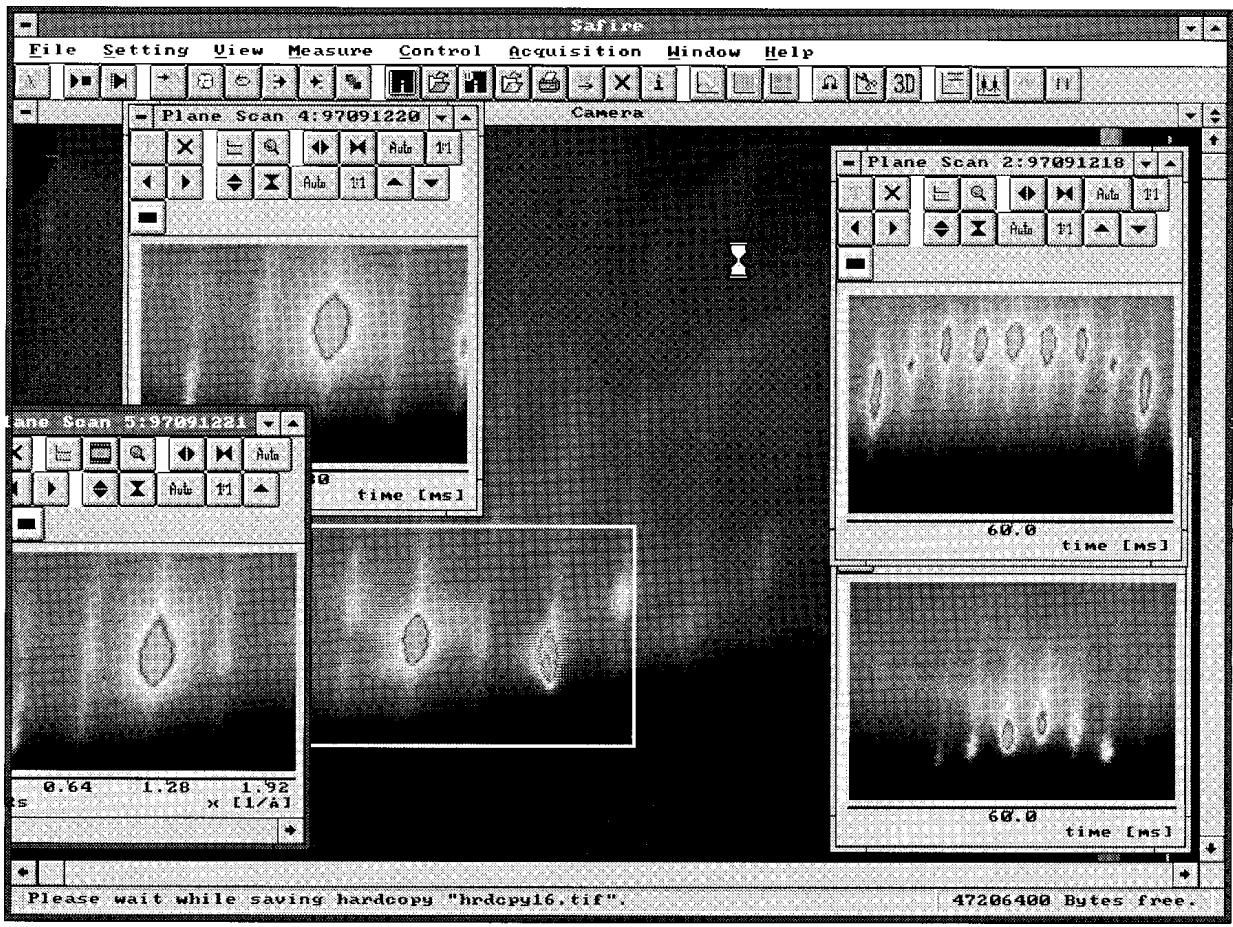

FIG. 1. Snapshot of RHEED patterns taken along the four $\langle 110\rangle$ azimuths during continuous rotation of the substrate. The source area of the data is indicated by a bright frame in the background image. The measurement was made at $580{ }^{\circ} \mathrm{C}$ on a GaAs $(001)$ surface reconstructed in the $\beta(2 \times 4)$ structure using 20 keV electrons. Left: [110] and [110] azimuths, right: [110] and [110] azimuths.

therefore allows a very detailed assessment of the reciprocal lattice. All the standard measurements like evolution of intensities, peak shapes, etc. can be performed with a maximum rate corresponding to the revolution frequency by subsequent analysis extracting data from the individual displays.

The triggered recording of images during rotation allows access to several discrete cuts of the reciprocal lattice. A complete scan of the accessible reciprocal space can be achieved by recording continuously during sample rotation. The basic diffraction geometry of RHEED is shown in Fig. 2(a). For any incidence angle $\theta$, the Ewald sphere construction requires both $k_{0}$ and $k^{\prime}$ to end on a reciprocal lattice rod a)

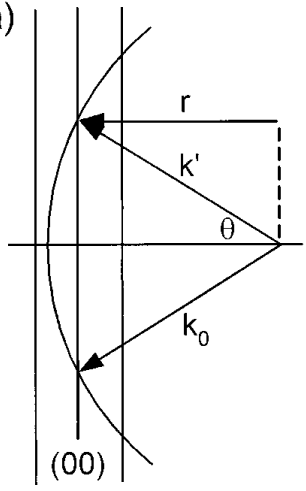

b)

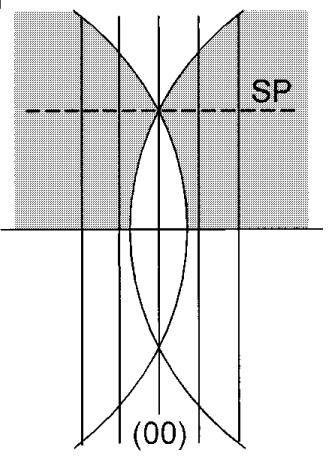

FIG. 2. Reciprocal space geometry for (a) static RHEED and (b) during rotation. The shaded area in (b), rotated around (00), represents the volume of reciprocal space accessible during a single sample rotation. The only surface parallel plane without a void in the center, the specular plane, intersects the specular spot and is indicated by a dashed line. for diffraction to occur. During sample rotation, the reciprocal lattice revolves around the $(00)$ rod, while the Ewald sphere stays fixed in space if we neglect wobble. The Ewald sphere therefore scans almost the entire upper half of reciprocal space as indicated by the shaded area in Fig. 2(b). The scanned volume is largest for the smallest incidence angles $\theta$, where the wave vector component perpendicular to the surface is smallest, ensuring high surface sensitivity. This allows us to reconstruct practically the entire upper half of reciprocal space during one sample revolution.

In a practical situation, this is not possible in real time due to the lack of capability of current computers. We therefore presently restrict ourselves to a plane parallel to the surface and choose the plane containing the specular spot, since it is the only plane that is continuous across the (00) rod. We denote this plane as the specular plane (SP). Its position is indicated in Fig. 2(b) by a dashed line. The measurement takes place by recording the intensities along a line parallel to the shadow edge through the specular spot as shown in Fig. 3(a). The specular plane is then constructed by plotting the lines bent with radius $r$ and the specular spot centered on (00) according to their azimuthal angle. This is schematically plotted in Fig. 3(b). The radius $r$ is given by $r=k_{0} \sin \theta$ given by the geometry in Fig. 2(a). As the rotation is completed, the lines add up to a complete cut through the reciprocal lattice at a distance $2 k_{0} \cos \theta$ from the origin of the reciprocal lattice. Such a cut is similar to a low-energy electron diffraction (LEED) pattern and represents the twodimensional (2D) symmetry of the surface reconstruction in a top view. By varying the incidence angle, a wide range of 

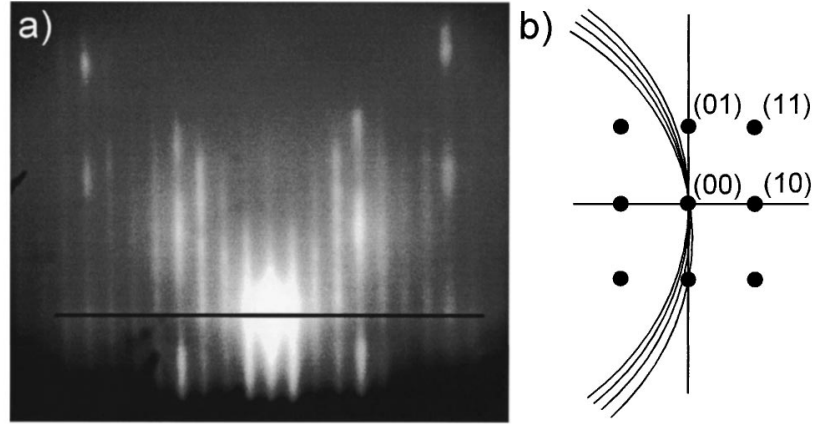

FIG. 3. Measurement geometry and top view of the azimuthal scan. Experimental conditions in (a) are similar to those in Fig. 1, close to the [110] azimuth. The intensity distribution in the specular plane is obtained by plotting the lines according to their azimuthal angle with the specular spot centered at (00) and radius $r$ (see Fig. 2).

wave vectors perpendicular to the surface can be measured, similar to a $I-V$ measurement in LEED.

\section{RESULTS}

To demonstrate the method, we have investigated the $\beta(2 \times 4)$ and $c(4 \times 4)$ surface reconstructions of $\mathrm{GaAs}(001)$. The accuracy in both cases was impeded by charging effects close to the RHEED screen leading to a distorted RHEED pattern and unsteady rotation that resulted from the gears not being driven precisely by a magnetically coupled feedthrough. Both factors contributed to a slight distortion of the processed scan. The results are shown in Fig. 4 for the $\beta(2 \times 4)$ and in Fig. 5 for the $c(4 \times 4)$ surface reconstructions, respectively. The rotation speed was approximately 3 $\mathrm{rpm}$. The intensity profiles were recorded at a rate of $50 \mathrm{~Hz}$, resulting in an angular resolution of about $0.4^{\circ}$. The inci-

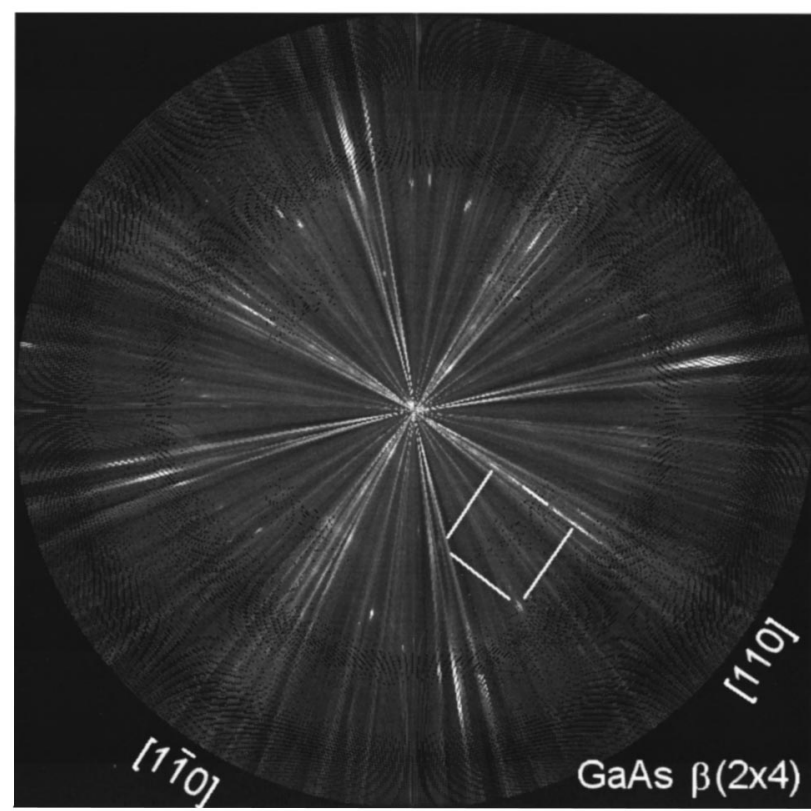

FIG. 4. Azimuthal scan of a GaAs $\beta(2 \times 4)$-reconstructed surface. The main azimuths and the surface unit cell are indicated.

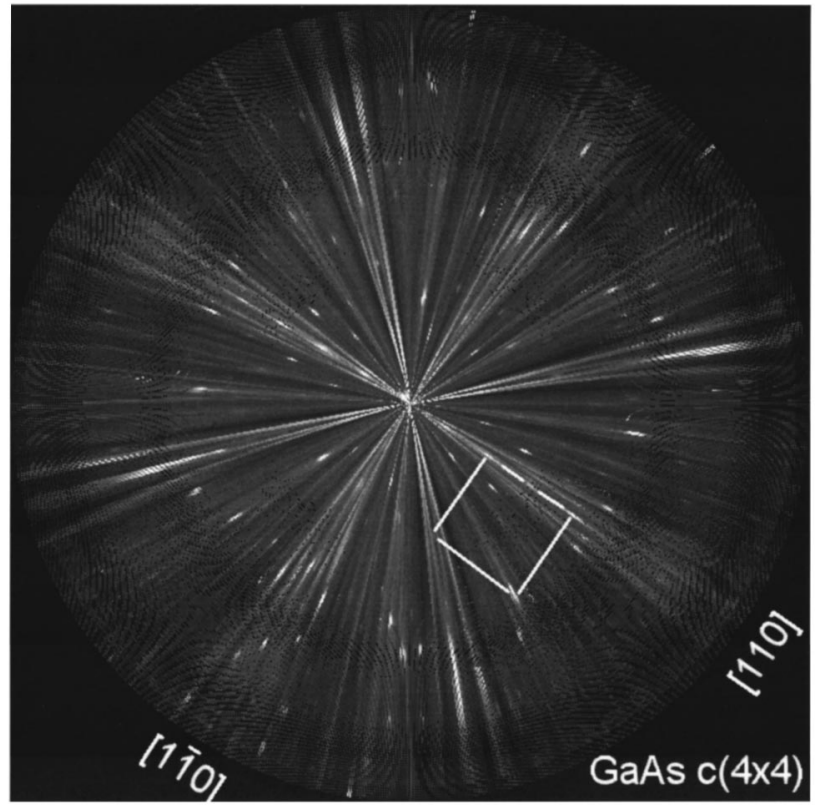

FIG. 5. Azimuthal scan of a GaAs $c(4 \times 4)$-reconstructed surface. The main azimuths and the surface unit cell are indicated.

dence angle $\theta$ was $1.5^{\circ}$ using $20 \mathrm{keV}$ electrons. In both images, the underlying $1 \times 1$ mesh of the surface reciprocal lattice is indicated by a white square. The scan spans more than six unit cells in each direction. This size is only limited by the electron energy (higher electron energy $E$ reduces the distance between the reciprocal lattice rods $\sim E^{-1 / 2}$ ) and the size of the RHEED screen. In our setup, this limit was approximately 16 unit cells for GaAs. The eight in-plane $\langle 001\rangle$ and $\langle 110\rangle$ azimuths can easily be identified by the adjacent high intensity radial streaks. These correspond to surface resonance conditions ${ }^{10-12}$ where the Laue circle tangentially touches reciprocal lattice rods, leading to increased reflectivity.

Spots in the azimuthal scan correspond to intersections of reciprocal lattice rods with the specular plane. The spots are of elliptic shape with the longer axis along the radial direction. The anisotropy is a direct measure of the different transfer widths $t$ (Ref. 13) of RHEED parallel and perpendicular to the beam that are not proportional to the normalized full width at half maximum (FWHM) of the spot in the respective direction:

$$
t=\frac{a_{i j} g_{i j}}{h},
$$

where $h$ denotes the FWHM of the spot and $a_{i j}$ and $g_{i j}$ are the real and reciprocal lattice constants in the direction the FWHM is measured. In Fig. 4 , the $\beta(2 \times 4)$ structure is clearly identified by the rows of closely spaced spots directed along [110]. This distinguishes it from the $c(4 \times 4)$ structure in Fig. 5 that exhibits a centered mesh. A display of the full 2D symmetry of the reciprocal lattice allows an immediate distinction, e.g., between $c(4 \times 4)$ and $(2 \times 2)$ structures to 
be made that is not evident from observation along the $\langle 110\rangle$ azimuths, where both would exhibit the same symmetry.

The $c(4 \times 4)$ pattern in Fig. 5 does not have a fourfold rotational symmetry. If we look, for example, at the spots in the row directly adjacent to the [110] direction, we observe high intensity on the second, third, and fifth spots from (00). In the corresponding row-along [1히, he third spot is very weak. This deviation from the fourfold, symmetry is present even on $(1 \times 1)$ reconstructed surfaces and allows identification of the two different azimuths even if the static RHEED patterns along both azimuths differ only very little. It is due to asymmetries in the structure like the orientation of dimers. Therefore, azimuthal scans are promising candidates to compare against theoretical models. The ease of acquisition together with the simple geometry allow the acquisition of highly accurate data. At the same time, the access to a large region of reciprocal space spanning many unit cells makes the comparison with models easier since many points can be fitted simultaneously. Since in the kinematical approximation the structure of reciprocal space far from the (00) rod contains the most detailed information about the atomic positions, ${ }^{14}$ even structure optimization seems feasible and can be expected to be more reliable than previous optimizations using data sets obtained from conventional RHEED scans. $^{15-18}$

The comparison of Figs. 4 and 5 suggests a reliable method for in situ control of surface reconstruction. If the growth process requires the surface to maintain a certain reconstruction, the respective spots in the azimuthal scan can be monitored during rotation. The two-dimensional nature of the measurement permits the simultaneous monitoring of many spots, resulting in increased accuracy and allowing consistency checks. In this way, a control algorithm could, for example, distinguish whether the desired surface reconstruction changes to the adjacent low- or high-temperature configuration if it controlled the sample temperature and change it accordingly.

\section{SUMMARY}

In this work, we presented new ways to access reciprocal space during substrate rotation. Both gated detection and azi- muthal scans provide direct access to the surface reconstruction in real time during growth, allowing in situ control of the growth front. Neither approach requires modifications to the MBE machine except for the generation of a trigger signal by the rotation motor and either is reliable enough to work on standard MBE machines. In addition to conventional recording techniques, azimuthal scans provide full $2 \mathrm{D}$ symmetry of the reciprocal lattice, a direct ratio of the transfer width of the instrument both parallel and perpendicular to the beam, and access to large portions of reciprocal space with a single scan. These properties make azimuthal scans ideal candidates for comparison with theoretical models of RHEED.

\section{ACKNOWLEDGMENTS}

The authors would like to thank C.-H. Kuo for the MBE growth. This work was supported by DARPA under Contract No. MDA972-95-1-0016.

${ }^{1}$ G. W. Turner and A. J. Isles, J. Vac. Sci. Technol. B 10, 1784 (1992). ${ }^{2}$ J. P. A. van der Wagt and J. S. Harris, Jr., J. Vac. Sci. Technol. B 12, 1236 (1994).

${ }^{3}$ D. A. Collins, G. O. Papa, and T. C. McGill, J. Vac. Sci. Technol. B 13, 1953 (1995).

${ }^{4}$ R. F. Kromann, R. N. Bicknell-Tassius, A. S. Brown, J. F. Dorsey, K. Lee, and G. May, J. Cryst. Growth 175/176, 334 (1997).

${ }^{5}$ G. W. Turner, B. A. Nechay, and S. J. Eglash, J. Vac. Sci. Technol. B 8, 283 (1990).

${ }^{6}$ J. J. Harris, B. A. Joyce, and P. J. Dobson, Surf. Sci. 103, L90 (1981).

${ }^{7}$ W. Braun, A. Trampert, L. Däweritz, and K. H. Ploog, Phys. Rev. B 55, 1689 (1997).

${ }^{8}$ S. Ino, Reflection High-Energy Electron Diffraction and Reflection Electron Imaging of Surfaces, edited by P. K. Larsen and P. J. Dobson (Plenum, New York, 1988), p. 3.

9"'Safire" by VTS Schwarz/FhG Erlangen.

${ }^{10}$ A. Ichimiya, K. Kambe, and G. Lehmpfuhl, J. Phys. Soc. Jpn. 49, 684 (1980).

${ }^{11}$ G. Meyer-Ehmsen, in Ref. 8, p. 99.

${ }^{12}$ L.-M. Peng, J. Cowley, and N. Yao, Ultramicroscopy 26, 189 (1988).

${ }^{13}$ M. Henzler, Appl. Surf. Sci. 11/12, 450 (1982).

${ }^{14}$ W. Braun, O. Brandt, M. Wassermeier, L. Däweritz, and K. H. Ploog, Appl. Surf. Sci. 104/105, 35 (1996).

${ }^{15}$ Y. Horio and A. Ichimiya, Surf. Sci. 219, 128 (1993).

${ }^{16}$ Y. Ma, S. Lordi, P. K. Larsen, and J. A. Eades, Surf. Sci. 289, 47 (1993).

${ }^{17}$ J. M. McCoy, U. Korte, P. A. Maksym, and G. Meyer-Ehmsen, Surf. Sci. 306, 247 (1994).

${ }^{18}$ Y. Ma, S. Lordi, P. K. Larsen, and J. A. Eades, Surf. Sci. 306, 252 (1994). 